\title{
Variations in Practice Patterns among Neurosurgeons and Orthopaedic Surgeons in the Management of Spinal Disorders
}

\author{
Manzar Hussain', Sadaf Nasir², Amber Moed ${ }^{3}$, Ghulam Murtaza ${ }^{4}$ \\ 'Section of Neurosurgery, Department of Surgery, Aga Khan University Hospital, Karachi, Pakistan \\ 'Liaquat National Hospital, Karachi, Pakistan \\ ${ }^{3}$ Department of Epidemiology and Biostatistics, Aga Khan Univerisity Hospital, Karachi, Pakistan \\ ${ }^{4}$ Department of Surgery, Aga Khan University Hospital, Karachi, Pakistan
}

Study Design: This is a case series.

Pupose: We wanted to identify variations in the practice patterns among neurosurgeons and orthopedic surgeons for the management of spinal disorders.

Overview of Literature: Spinal disorders are common in the clinical practice of both neurosurgeons and orthopedic surgeons. It has been observed that despite the availability of various guidelines, there is lack of consensus among surgeons about the management of various disorders.

Methods: A questionnaire was distributed, either directly or via e-mail, to the both the neurosurgeons and orthopedic surgeons who worked at 5 tertiary care centers within a single region of Korea. The surgeons were working either in private practice or in academic institutions. The details of the questionnaire included demographic details and the specialty (orthopedic/neurosurgeon). The surgeons were classified according to the level of experience as up to 5 years, 6-10 years and $>10$ years. Questions were asked about the approach to lumbar discectomy (fragmentectomy or aggressive disc removal), using steroids for treating discitis, the fusion preference for spondylolisthesis, the role of an orthosis after fusion, the preferred surgical approach for spinal stenosis, the operative approach for spinal trauma (early within 72 hours or late $>72$ hours) and the role of surgery in complete spinal cord injury. The data was analyzed using SPSS ver 16. $p$-values $<0.05$ were considered to be significant.

Results: Of the 30 surgeons who completed the questionnaire, 20 were neurosurgeons and 10 were orthopedic surgeons. Statistically significant differences were observed for the management of spinal stenosis, spondylolisthesis, using an orthosis after fusion, the type of lumbar discectomy and the value of surgical intervention after complete spinal cord injury.

Conclusions: Our results suggest that there continues to exist a statistically significant lack of consensus among neurosurgeons and orthopedic spine surgeons when considering using an orthosis after fusion, the type of discectomy and the value of intervention after complete spinal injury.

Key Words: Orthopedic surgeons, Neurosurgeons, Spinal trauma, Spinal stenosis, Complete cord injury

\section{Introduction}

Spinal disorders are an important part of the clinical prac- tice of neurosurgeons and orthopedic surgeons. The majority of spinal pathologies are treated by neurosurgeons or some orthopedic surgeons in modern medical practice. There continues to exist a lack of consensus among sur-

Received Feb 21, 2011; 1st Revised Apr 9, 2011; Accepted Apr 25, 2011

Corresponding author: Manzar Hussain, MBBS, FCPS

Flat No.a-30, Erum Garden, Block 13/d, Near Hassan Square, Gulshan-e-iqbal, Karachi 75300, Pakistan

Tel:+92-343-2025927, E-mail: manzary2k@yahoo.com 
geons as to the optimum management and appropriateness of multiple aspects of specific operative and non-operative interventions for various spinal disorders, including degenerative spinal disorders, with special reference to spinal trauma [1]. A concerted effort was made in 2004 to solve this issue [2]. The researchers concluded that considerable agreement existed for the majority of clinical decision making. Despite this, we continue to observe differences among various surgeons who deal with spinal disorders [2]. This issue is especially important in 3rd world countries where uniformity in clinical practice might help in reducing costs. This study was designed to identify the differences in the practice patterns among the orthopedic and neurosurgeons who treat patients with spinal disorders in our local Korean medical hospitals. Knowing these differences will help us to identify the areas of weakness and possibly come up with algorithms that are acceptable to both neurosurgeons and orthopedic surgeons.

\section{Materials and Methods}

A questionnaire was prepared and it was distributed to both neurosurgeons and orthopedic surgeons who worked at 5 tertiary care centers within a single region of Korea. The surgeons were working either in private practice or in academic institutions. The surgeons were either approached directly or their responses were received through e-mail. All the surgeons were either local fellowship trained or foreign trained. The details included in the questionnaire were demographic details and specialty (orthopedic/neurosurgeon). The surgeons were classified according to their level of experience with dealing with various spinal disorders: up to 5 years, 6-10 years and $>10$ years. The surgeons were also classified on the basis of age. Most of the surgeons were also involved in teaching residents and medical students. Questions were asked about the approach to lumbar discectomy (i.e., fragmentectomy or aggressive disc removal), the use of steroids in discitis, the fusion preference in spondylolisthesis, the role of an orthosis after fusion, the preferred surgical approach for spinal stenosis, the operative approach for spinal trauma (early within 72 hours or late $>72$ hours) and the role of surgery for complete spinal cord injury with taking into account various factors like mechanical instability in addition to neurological deficits. All these questions were chosen because these are the common pathologies and issues encountered in our clinical practice. The data was analyzed using SPSS ver. 16 (SPSS Inc., Chicago, IL, USA). $p$-values $<0.05$ were deemed statistically significant.

\section{Results}

All the surgeons were contacted and the questionnaire was filled out either by direct contact or through e-mail. Seventy percent of the surgeons were associated with a teaching hospital, while $30 \%$ were in private practice. Of the 30 surgeons enrolled in the study 19 were $\leq 40$ years of age, 8 were between 41-50 and 3 were $>50$ years of age. All of the surgeons were male, whether they were orthopedic surgeons or neurosurgeons. Of the 30 surgeons, 10 were orthopedic surgeons and 20 were neurosurgeons. This is because spine care is predominantly provided by the neurosurgeons. The surgeons were divided into 3 groups according to the level of their experience. Group 1 included surgeons with up to 5 years' experience. The 2nd group consisted of surgeons with experience between 6-10 years. The 3rd group consisted of surgeons with more than 10 years' experience. Group 1 included 7 surgeons, group 2 included 15 surgeons and group 3 included 8 surgeons. The demographic details are given in Table 1. The responses of the surgeons are summarized in Table 2.

Our results showed that there were significant disagreements between the neurosurgeons and orthopaedic surgeons for the questions about the management of various spinal disorders. When asked about the use of orthoses, all the neurosurgeons opted for some kind of post-operative orthosis, while only $50 \%$ of the orthopedic surgeons opted for a post-operative orthosis $(p$-value $=0.003)$. When asked about aggressive discectomy $50 \%$ of the neurosurgeons were in favor of this, while all the orthopedic surgeons were in favor of aggressive discectomy ( $p$-value $=0.01$ ). When the surgeons were asked about their opinion regarding sur-

Table 1. Demographic details

\begin{tabular}{lll}
\hline \hline & Orthopedic surgeons $(\mathrm{n}=10)$ & Neurosurgeons $(\mathrm{n}=20)$ \\
\hline Gender & Males & Males \\
Mean age \pm SD (range, yr) & $43.9 \pm 5.237(38-52)$ & $41.95 \pm 5.44(38-57)$ \\
\hline
\end{tabular}

SD: Standard deviation. 
Table 2. Surgeons responses to the questionnaire (only the yes response is given)

\begin{tabular}{|c|c|c|c|}
\hline & $\begin{array}{l}\text { Neurosurgeons } \\
\quad(\mathrm{n}=20)\end{array}$ & $\begin{array}{l}\text { Orthopedic surgeons } \\
\qquad(\mathrm{n}=10)\end{array}$ & $\begin{array}{l}\text { Statistically significant differences between } \\
\text { neurosurgeon and orthopedic surgeons ( } p \text {-value) }\end{array}$ \\
\hline \multicolumn{4}{|l|}{ Spinal stenosis } \\
\hline Interlaminar decompression & $4(20)$ & & \\
\hline Decompressive laminectomy & $16(80)$ & $10(100)$ & \\
\hline \multicolumn{4}{|c|}{ Spondylolisthesis + stenosis (fixed deformity) } \\
\hline Instrumented fusion & $14(70)$ & $10(100)$ & \\
\hline Non-instrumented fusion & $6(30)$ & & \\
\hline Orthosis after fusion & $20(100)$ & $5(50)$ & 0.003 \\
\hline Steroids in discitis & $14(70)$ & $5(50)$ & \\
\hline \multicolumn{4}{|l|}{ Lumbar discectomy } \\
\hline Fragmentectomy & $10(50)$ & - & \\
\hline Aggressive disc removal & $10(50)$ & $10(100)$ & 0.01 \\
\hline \multicolumn{4}{|l|}{ Intervention in spinal trauma } \\
\hline Early (within $72 \mathrm{hr}$ ) & - & - & \\
\hline Late $(>72 \mathrm{hr})$ & $20(100)$ & $10(100)$ & \\
\hline \multicolumn{4}{|l|}{ Intervention in spinal injury } \\
\hline Complete injury & $6(30)$ & $8(80)$ & 0.02 \\
\hline
\end{tabular}

Values are presented as number of surgeons (\%).

Only statistically significant differences are mentioned. $p$-value $<0.05$ was taken to be significant.

gical intervention for patients with complete cord injury, only $30 \%$ of the neurosurgeons were in favor of any surgical intervention, while about $80 \%$ of the orthopedic surgeons were in favor of some kind of surgical intervention $(p$-value $=0.02)$.

\section{Discussion}

In the present era it is expected that most of the spinal pathologies will be managed by spinal surgeons with the firm understanding that a clear set of guidelines and management protocols will be followed for these pathologies. The medical literature is full of treatment algorithms for the management of common spinal disorders, yet there still remains significant controversy about the management of various spinal disorders [3-10].

These differences are not only within a specialty, but they also extend between the specialties. In addition to this, these differences are not only restricted to trauma, but they also include common spinal degenerative issues. The differences include both operative and non-operative protocols [11-16]. It has been observed that at times family and social pressures dictate the surgeons' opinions rather than pure science [17]. Because of their different ways of training, both neurosurgeons and orthopedic surgeons have developed their own classifications for the management of common spinal disorders $[18,19]$. Although differences in practice patterns exist between neurosurgeons and orthopaedic surgeons, there is also some common ground [20].

The question arises: Why are there such discrepancies in management protocols? A review of the literature suggests that most of the published medical literature is in the form of class III evidence. Such reviews are more susceptible to bias than the more reliable prospective studies, and these biases potentially influence patient recruitment, patient treatment, the available data and the interpretation of results in a negative way. This is probably one of the reasons why various spine surgeons interpret these studies differently. It must also be remembered that for many clinical scenarios there is more than one suitable treatment option. In these cases, additional variables such as training, experience, technical expertise, location and the available resources may lead a surgeon to prefer certain treatment options over others. One important reason for these differences is lack of uniform training among orthopedic surgeons and neurosurgeons. This fact has also been highlighted by other investigators [21].

It must be kept in mind that most of the care for spinal pathologies is provided by neurosurgeons. There can be number of possible explanations for these differences in the care of relatively routine spine pathologies in our study. We believe that the differences among training and clinical experience are probably the most obvious explanations and the failure to appreciate the recent changes in the manage- 
ment of these disorders may also be a factor. The detailed explanation of these factors was out of the scope of this study.

This study has highlighted an area of management of spinal disorders where investment in education may lead to significant improvement. Structured training and exchange programs amongst various residency programs may help minimize this discrepancy.

We believe that the strength of our study is that we included surgeons with various experience and both orthopedic surgeons and neurosurgeons. Another strength of our study is that we included those questions in our questionnaire that are routinely addressed by surgeons who treat patients with various spinal disorders. This study provided these surgeons the opportunity to express various aspects of their patient management regarding common spinal issues.

This study has a number of important limitations. The surgeons were limited to only one region of Korea. The results probably do not accurately represent the attitude of all practicing neuro/orthopedic surgeons and so the results serve more as an initial effort towards exploring this issue. The study did not assess whether the background education of these surgeons (neurosurgeons or orthopedic surgeons) influenced their practice management. It must also be recognized that various social/cultural factors were not investigated and these may play a significant role for the operative and perioperative management of these patients. Another limitation of this study was that the study did not look into how these diverse practice patterns affected the patients' outcomes.

\section{Conclusions}

Our results suggest that there continues to exist a statistically significant lack of consensus among neurosurgeons and orthopedic spine surgeons when considering using an orthosis after fusion, the type of discectomy and whether to perform intervention for treating complete spinal injury.

\section{REFERENCES}

1. Lenehan B, Dvorak MF, Madrazo I, Yukawa Y, Fisher CG. Diversity and commonalities in the care of spine trauma internationally. Spine (Phila Pa 1976) 2010;35(21 Suppl):S174-9.

2. Grauer JN, Vaccaro AR, Beiner JM, et al. Similarities and differences in the treatment of spine trauma between surgi- cal specialties and location of practice. Spine (Phila $\mathrm{Pa}$ 1976) 2004;29:685-96.

3. Elgafy H, Dvorak MF, Vaccaro AR, Ebraheim N. Treatment of displaced type II odontoid fractures in elderly patients. Am J Orthop (Belle Mead NJ) 2009;38:410-6.

4. Grauer JN, Vaccaro AR, Lee JY, et al. The timing and influence of MRI on the management of patients with cervical facet dislocations remains highly variable: a survey of members of the Spine Trauma Study Group. J Spinal Disord Tech 2009;22:96-9.

5. Lee JY, Nassr A, Eck JC, Vaccaro AR. Controversies in the treatment of cervical spine dislocations. Spine $\mathrm{J}$ 2009;9:418-23.

6. Nassr A, Lee JY, Dvorak MF, et al. Variations in surgical treatment of cervical facet dislocations. Spine (Phila Pa 1976) 2008;33:E188-93.

7. Smith HE, Vaccaro AR, Maltenfort M, et al. Trends in surgical management for type II odontoid fracture: 20 years of experience at a regional spinal cord injury center. Orthopedics 2008;31:650.

8. Stadhouder A, Buskens E, de Klerk LW, et al. Traumatic thoracic and lumbar spinal fractures: operative or nonoperative treatment: comparison of two treatment strategies by means of surgeon equipoise. Spine (Phila Pa 1976) 2008;33:1006-17.

9. Zdeblick TA, Sasso RC, Vaccaro AR, Chapman JR, Harris MB. Surgical treatment of thoracolumbar fractures. Instr Course Lect 2009;58:639-44.

10. Fisher CG, Noonan VK, Dvorak MF. Changing face of spine trauma care in North America. Spine (Phila Pa 1976) 2006;31(11 Suppl):S2-8

11. Irwin ZN, Hilibrand A, Gustavel M, et al. Variation in surgical decision making for degenerative spinal disorders. Part I: lumbar spine. Spine (Phila Pa 1976) 2005;30:220813.

12. Dipaola CP, Bible JE, Biswas D, Dipaola M, Grauer JN, Rechtine GR. Survey of spine surgeons on attitudes regarding osteoporosis and osteomalacia screening and treatment for fractures, fusion surgery, and pseudoarthrosis. Spine J 2009;9:537-44

13. Glaser JA, Jaworski BA, Cuddy BG, et al. Variation in surgical opinion regarding management of selected cervical spine injuries: a preliminary study. Spine (Phila Pa 1976) 1998;23:975-82.

14. Irwin ZN, Hilibrand A, Gustavel M, et al. Variation in surgical decision making for degenerative spinal disorders. Part II: cervical spine. Spine (Phila Pa 1976) 2005;30: 
2214-9.

15. Ploumis A, Ponnappan RK, Sarbello J, et al. Thromboprophylaxis in traumatic and elective spinal surgery: analysis of questionnaire response and current practice of spine trauma surgeons. Spine (Phila Pa 1976) 2010;35:323-9.

16. Pickett GE, Van Soelen J, Duggal N. Controversies in cervical discectomy and fusion: practice patterns among Canadian surgeons. Can J Neurol Sci 2004;31:478-83.

17. Bederman SS, Mahomed NN, Kreder HJ, McIsaac WJ, Coyte PC, Wright JG. In the eye of the beholder: preferences of patients, family physicians, and surgeons for lumbar spinal surgery. Spine (Phila Pa 1976) 2010;35:108-15.

18. Arnold PM, Brodke DS, Rampersaud YR, et al. Differences between neurosurgeons and orthopedic surgeons in classifying cervical dislocation injuries and making assessment and treatment decisions: a multicenter reliability study. Am J Orthop (Belle Mead NJ) 2009;38:E156-61.

19. Raja Rampersaud Y, Fisher C, Wilsey J, et al. Agreement between orthopedic surgeons and neurosurgeons regarding a new algorithm for the treatment of thoracolumbar injuries: a multicenter reliability study. J Spinal Disord Tech 2006;19:477-82.

20. Magit DP, Hilibrand AS, Kirk J, et al. Questionnaire study of neuromonitoring availability and usage for spine surgery. J Spinal Disord Tech 2007;20:282-9.

21. Dvorak MF, Collins JB, Murnaghan L, et al. Confidence in spine training among senior neurosurgical and orthopedic residents. Spine (Phila Pa 1976) 2006;31:831-7. 